\title{
Formulation of Impulsive Differential Equations with Time-Dependent Continuous Delay
}

\author{
Ita Micah Esuabana ${ }^{1}$, Ubon Akpan Abasiekwere ${ }^{2}$ \\ ${ }^{1}$ Department of Mathematics, Faculty of Science, University of Calabar, Calabar, Nigeria \\ ${ }^{2}$ Department of Mathematics and Statistics, Faculty of Science, University of Uyo, Uyo, Nigeria
}

Email address:

esuabanaita@gmail.com (I. M. Esuabana), ubeeservices@yahoo.com (U. A. Abasiekwere)

\section{To cite this article:}

Ita Micah Esuabana, Ubon Akpan Abasiekwere. Formulation of Impulsive Differential Equations with Time-Dependent Continuous Delay. American Journal of Applied Mathematics. Vol. 6, No. 4, 2018, pp. 135-141. doi: 10.11648/j.ajam.20180604.11

Received: September 10, 2018; Accepted: October 9, 2018; Published: October 31, 2018

\begin{abstract}
Research in impulsive delay differential equations has been undergoing some exciting growth in recent times. This to a large extent can be attributed to the quest by mathematicians in particular and the science community as a whole to unveil nature the way it truly is. The realization that differential equations, in general, and indeed impulsive delay differential equations are very important models for describing the true state of several real-life processes/phenomena may have been the tunic. One can attest that in most human processes or natural phenomena, the present state is most often affected significantly by their past state and those that were thought of as continuous may indeed undergo abrupt change at several points or even be stochastic. In this study, a special strictly ascending continuous delay is constructed for a class of system of impulsive differential equations. It is demonstrated that even though the dynamics of the system and the delay have ideal continuity properties, the right side may not even have limits at some points due to the impact of past impulses in the present. The integral equivalence of the formulated system of equations is also obtained via a scheme similar to that of Perron by making use of certain assumptions.
\end{abstract}

Keywords: Impulsive, Differential Equation, Continuous Delay, Integral Equivalence

\section{Introduction and Statement of Problem}

The theory of impulsive delay differential equations (IDE) is based on the behaviour of processes or phenomena which undergo abrupt changes in their state and past events affect the current behaviour (delay). These kinds of processes are best described by coupled systems of differential equations, either starting with delay differential equations and adding impulses or starting with impulsive differential equations and adding delay arguments. Whichever is the case, a coupled problem is obtained and the structure is radically changed. Several of the properties of solutions in ordinary, delay, or impulsive differential equations are no longer sustained.

The theory of impulsive delay differential equations have been relatively less developed because of significant technical and theoretical difficulties, and as such only a few pieces of literature are available [1-2]. However, interest is on the increase largely due to the fact that a lot of everyday phenomena in Sciences, Economics, Engineering, Space sciences, and control systems are modeled by impulsive delay differential equations [1, 3-5]. In particular, Ballinger's Ph.D thesis and his subsequent work provide a good working tool for further research work in this area, especially, as it relates to existence, uniqueness, boundedness, continuation, and stability of solutions of Impulsive Delay Differential Equations (IDDE) [3]. This happens to be a fusion of two areas - Delay Differential Equations, and Impulsive Differential Equations. Several evolution processes in Sciences, Engineering, Technology, Economics, etc., are modeled by impulsive differential equations with delays [1, 3-6].

In ordinary or delay differential equations, the solutions are continuously differentiable, at least once or more, whereas impulsive differential equations possess non-continuous (piecewise continuous) solutions. Intuitively, it is expected that the coupled problems of impulsive delay differential equations should possess piecewise continuously differentiable solutions. To some extent, our intuition may be true if the delays are discrete. When continuous delays interplay with impulses, the 
story may become significantly different. This surge in the number of discontinuous points creates several problems as it pertains to existence, stability/instability of solutions, just to mention a few. Since the continuity properties of the solutions play fundamental roles in the analysis of their behaviour, the techniques used to handle the solutions of impulsive delay differential equations are basically different from those of Ordinary Differential Equations, Impulsive Differential Equations and Delay Differential Equations. However, some basic concepts are still valid.

In this study, a system of first-order impulsive differential equations with continuous time-dependent delays and fixed moments of impulse is formulated. Some recent results in impulsive delay differential equations with constant impulsive jumps can be seen in [7-14].

\section{Preliminaries}

The theory of impulsive systems was developed not long ago as an independent area of mathematical analysis. The development arose out of curiosity to develop a mathematical framework that truly describes physical and biological processes as they occur in nature. Prior to this noble development, scientists had often made an underlying assumption that the behaviour of physical and biological systems described by ordinary differential equations is continuous and integrable in some sense. It was observed that the state of a system is susceptible to changes, and in some processes, these changes are often characterized by shorttime perturbations (impulses) whose durations are negligible when compared with the total duration of their entry time evolution [15, 3, 16-18].

IDEs are adequate mathematical models for the description of evolution processes characterized by the combination of continuous and jump changes of their state. For the continuous change of such processes, ordinary differential equations are used, while the moments and the magnitude of the jumps are given by the jump conditions [15, 19-21]. Impulsive systems are systems whose states are characterized by small perturbations (impulses) in the form of jumps [22-24].

These equations are classified into two categories: those with fixed moments of impulsive effects (moments of jumps are previously fixed), and those with unfixed moments of impulsive effects (moments of jump occur when certain space-time relations are satisfied) $[15,18,20,25]$.

IDEs are usually defined by a pair of equations - an ordinary differential equation to be satisfied during the continuous portion of the evolution, and difference equations defining the change of state at the discrete impulsive points. This is the main formulation of early scholars such as Bainov, Simeonov, Lakshminkatham, Gopalsamy, Zhang, among others. Solutions are usually considered to be piecewise continuously differentiable functions with discontinuities occurring at the impulsive times [3, 26].

Impulsive differential equations with fixed moments of impulsive effects have the form:

$$
\left\{\begin{array}{c}
x^{\prime}(t)=f(t, x(t)), \quad \forall t \in T \backslash S \\
\Delta x\left(t_{k}\right)=f\left(t_{k}, x\left(t_{k}\right)\right), \quad \forall t_{k} \in S,
\end{array}\right.
$$

where $T \subset R,(t, x) \in \Omega \subset R \times R^{n}$ and the real numerical sequence $S=\left\{t_{k}\right\}_{k=1}^{\infty}$ increases and has no finite accumulation point. In the case of unfixed moments of impulsive effects, the impulse points may be time and state dependent. That is, $t_{k}:=t_{k}(t, x(t))$. When the function $t_{k}$ depends on the state of the given system, it is said to have impulses at variable times. This is reflected in the fact that different solutions will tend to undergo impulses at different times. However, if the functions $t_{k}$ are all constants, the system is said to have impulses at fixed times which implies that all solutions undergo impulse actions at the same time.

It is observed that the question of the existence of solutions of the system is non-trivial when impulses occur at variable times. The precise notion of what a solution is must be carefully stated. It is fairly clear that solutions should be piecewise continuous and in fact piecewise continuously differentiable (or piecewise absolutely differentiable when considering generalized types of solutions). A solution will undergo simple jump discontinuity when it intersects impulse hyper-surfaces. Even after focusing on a particular class of relations $t(s, x(s))=0$ given by impulse hyper-surfaces, impulsive differential equations still exhibit some unusual behaviour [3]. In this study, focus will be placed only on those equations with fixed moments of impulse effects.

$\mathrm{Be}$ as it may, to obtain or discuss the solution of an impulsive differential equation, certain peculiarities of the model must be taken into cognizance. It assume that for $t \in T \mid S$, the solution $x(t)$ of the earlier stated equation is determined by the ordinary differential equation $x^{\prime}(t)=f(t, x(t))$. For $t \in S$, a change by jump of the solution $x(t)$ occurs so that $x\left(t_{k}^{-}\right)=x\left(t_{k}\right)$ and $x\left(t_{k}^{+}\right)=x\left(t_{k}\right)+\Delta x\left(t_{k}\right)$ $=x\left(t_{k}\right)+f\left(t_{k}, x\left(t_{k}\right)\right)$. After the jump, at the moment $t=t_{k}$, the solution $x(t)$ of the system coincides with the solution $y(t)$ of the initial value problem [15]:

$$
\left\{\begin{array}{cl}
y^{\prime}(t)=f(t, y(t)), & t_{k}<t \leq t_{k+1} \\
\Delta y\left(t_{k}\right)=x\left(t_{k}^{+}\right), & t=t_{k} \in S .
\end{array}\right.
$$

This simply means that after the jump at $t=t_{k}$, a new function $y(t)$ takes over control from $x(t)$.

Let $T \subset R$ be a set of time points and let our processes take place in $R^{n}$. Also, let these processes be described by $x: T \rightarrow R^{n}$ state functions, assuming that they may be influenced by past events defined by delay functions $h_{j}: T \rightarrow R^{+}$. The properties of these functions will be specified later as progress is made.

Now, let $\bar{g}:=\left(g_{1}, g_{2}, \ldots, g_{m}\right), \hat{g}:=(g, g, \ldots, g) \in R^{m}$ and $\hat{f} \circ \bar{x}:=\left(f\left(x_{1}\right), f\left(x_{2}\right), \ldots, f\left(x_{m}\right)\right)$ it then follows that: 


$$
f\left(t, x(t), x\left(t-h_{1}(t)\right), x\left(t-h_{2}(t)\right), \ldots \ldots, x\left(t-h_{m}(t)\right)\right)=f(t, x(t), \hat{x} \circ(\hat{t}-\bar{h} \circ \hat{t})) .
$$

In the course of this work, it shall be assumed that $(a, b):=T \subset R$ is a non-empty open subset of $R$. For asymptotic investigation, at least $b=\infty$ is assumed. Let $S:=\left\{t_{k}\right\}_{k=1}^{\infty}$ or $S:=\left\{t_{k}\right\}_{k=-\infty}^{\infty}$ be an increasing sequence of numbers (to be referred to as impulse times or points) with at most two condensation points. Let $D^{*}:=S \times R^{n}$ and $D:=T \times R^{(m+1) \times n}$. Also, let $f: D \rightarrow R^{n}$ and $f^{*}: D^{*} \rightarrow R^{n}$ be continuous functions fulfilling Lipschitz condition in $x \in D$ and $x \in D^{*}$, respectively, at each fixed $t \in T$. Let $h_{i}: T \rightarrow R^{+}$ be continuous ascending delay function such that $h_{i}(t) \leq t-a, \forall t \in T, \quad \forall 1 \leq i \leq m$. Then from the notation in equation (1), a system of first-order impulsive differential equations with continuous delay is of the form:

$$
\left\{\begin{array}{l}
x^{\prime}(t)=f\left(t, x(t), x\left(t-h_{l}(t)\right), \ldots, x\left(t-h_{m}(t)\right)\right), \forall t \in T \backslash S \\
\Delta x\left(t_{k}\right)=x\left(t_{k}+0\right)-x\left(t_{k}-0\right)=f^{*}\left(t_{k}, x\left(t_{k}\right)\right), \forall t_{k} \in S .
\end{array}\right.
$$

From the notation in (1), it can be written in a more compact form as:

$$
\left\{\begin{array}{l}
x^{\prime}(t)=f(t, x(t), x \circ(\hat{t}-\bar{h} \circ \hat{t})), \quad \forall t \in T \mid S \\
\Delta x\left(t_{k}\right)=f^{*}\left(t_{k}, x\left(t_{k}\right)\right), \quad \forall t_{k} \in S .
\end{array}\right.
$$

Let $f: \Omega \rightarrow R^{n}, \Omega \subset T \times R^{n}$ be continuous and $x$ at least once differentiable. Let $h_{j} \in C(t)$ be continuous delay functions, $1 \leq j \leq m$ and $\bar{h} \circ \hat{t}=\left(h_{l}(t), h_{2}(t), \ldots, h_{m}(t)\right)$ be a continuous delay vector, then $r=\sup _{t \in(a, b)} \max _{1 \leq j \leq m}\left\{h_{j}(t)\right\}$ is called the delay constant (where the delays are discrete, that is, $r=\max _{l \leq j \leq m}\left\{h_{j}\right\}$ ).

Let equation (2) or equation (3) be given subject to the initial or history function

$$
x(t)=\varphi(t), t_{0}-r \leq t \leq t_{0},
$$

where $x\left(t_{k}\right)=x\left(t_{k}-0\right)$ and $f^{*}\left(t_{k}, x\left(t_{k}\right)\right)$ prescribes the jump at each impulse point $t_{k} \in S$, then equation (2) or (3) is called a first-order impulsive delayed differential equation with continuous delays. Subject to equation (4) it is called an initial value or function problem.

Let $A$ and $B$ be real $n$ by $n$ matrix functions with components in $C(a, b)$; let $\vec{g}$ be a vector with $n$ components in $C(a, b)$ and $\tilde{A}_{k}$ be an $n$ by $n$ matrix function on $S$, then a system of linear impulsive differential equation with continuous delays is defined as:

$$
\left\{\begin{array}{l}
x^{\prime}(t)=A(t)+B(t) \hat{x} \circ(\hat{t}-\bar{h} \hat{t})+\vec{g}, \forall t \in T \mid S \\
\Delta x\left(t_{k}\right)=\tilde{A}_{k} x\left(t_{k}\right), \forall t_{k} \in S .
\end{array}\right.
$$

If $\vec{g}$ is identically zero, equation (5) is called a homogeneous equation and is given by:

$$
\left\{\begin{array}{l}
x^{\prime}(t)=A(t) x(t)+B(t) \hat{x} \circ(\hat{t}-\bar{h} \hat{t}), \forall t \in T \mid S \\
\Delta x\left(t_{k}\right)=\tilde{A}_{k} x\left(t_{k}\right), \forall t_{k} \in S .
\end{array}\right.
$$

\section{Main Results}

Consider the system of equations given by

$$
\left\{\begin{array}{l}
x^{\prime}(t)=f(t, x(t), x(t-h(t))), \forall t \in T \mid S \\
\Delta x\left(t_{k}\right)=f^{*}\left(t_{k}, x\left(t_{k}\right)\right), \forall t_{k} \in S
\end{array}\right.
$$

which is similar to the system (6). This system assumes that for $t \in T \mid S$, the solution $x(t)$ is determined by the delay differential equation $x^{\prime}(t)=f(t, x(t), x(\hat{t}-\bar{h} \circ(\hat{t})))$ and for $t \in S$, a change by jump of the solution $x(t)$ occurs so that $x\left(t_{k}^{-}\right)=x\left(t_{k}\right)$ and $x\left(t_{k}^{+}\right)=x\left(t_{k}\right)+f^{*}\left(t_{k}, x\left(t_{k}\right)\right)[1-5]$.

Here, a special strictly ascending continuous delay for the system of equations in (6) is constructed.

The construction will take several steps as shown below:

Step 0: Let $\rho \in\left(t_{k}, t_{k}+1\right)$ for a fixed $k \in Z$. It is assumed here that $\left(t_{k}, t_{k+1}\right) \subset(\rho-\pi, \rho+\pi)$, otherwise, $\left(t_{k}, t_{k+1}\right)$ will be replaced by $\left(\alpha_{k}, \alpha_{k+1}\right)=\left(t_{k}, t_{k+1}\right) \cap(\rho-\pi, \rho+\pi)$. Moreover, let $t_{s} \in S, t_{s}<t_{k}$ be an impulse point in the past.

Step 1: Let $\varphi_{0}:\left(t_{k}, t_{k+1}\right) \rightarrow R^{+}$be defined by

$$
\varphi_{0}(t):=\left\{\begin{array}{cc}
\rho \in\left(t_{k}, t_{k+1}\right), & \varphi_{0}(\rho):=\sin \frac{1}{|t-\rho|} \quad \forall t \neq \rho \\
0, & \text { otherwise }
\end{array}\right.
$$

Remark 3.1. $\varphi_{0}$ has roots at $t=\rho \pm \frac{1}{k \pi}, \quad k \in N$.

Step 2: Let $\varphi_{1}(t):=|t-\rho|^{3} \varphi_{0}(t), \quad \forall t, \rho \in\left(t_{k}, t_{k+1}\right)$. Then its derivative is:

$$
\frac{d \varphi_{1}(t)}{d t}=\left\{\begin{array}{cc}
3|t-\rho|^{2} \varphi_{0}(t)-|t-\rho| \cos \frac{1}{|t-\rho|}, \quad t \neq \rho \\
0 & \text { otherwise }
\end{array}\right.
$$

$\forall t, \rho \in\left(t_{k}, t_{k+1}\right)$, which is bounded by $3\left(t_{k+1}-t_{k}\right)^{2}$.

Remark 3.2 In addition to Remark 3.1, $\varphi_{1}$ is continuously differentiable in $\left(t_{k}, t_{k+1}\right)$.

Step 3: Let us consider another function:

$$
\varphi_{2}(t)=\left(t-t_{k}\right)^{2}\left(t-t_{k+1}\right)^{2} \varphi_{1}(t), \quad \forall t \in\left(t_{k}, t_{k+1}\right) .
$$

This is continuously differentiable in $\left(t_{k}, t_{k+1}\right)$, its 
derivative is 0 at $t_{k}$ and $t_{k+1}$, and hence, it has a continuously differentiable extension to $T$.

Remark 3.3 In addition to Remark 3.2, $\varphi_{2}(t)$ fulfils $\varphi_{2}\left(t_{k}\right)=\varphi_{2^{\prime}}\left(t_{k}\right)=\varphi_{2}\left(t_{k+1}\right)=\varphi_{2^{\prime}}\left(t_{k+1}\right)=0$. Hence

$$
\varphi(t)= \begin{cases}\varphi_{2}(t), & t \in\left(t_{k}, t_{k+1}\right) \\ 0, & \text { otherwise }\end{cases}
$$

is a continuously differentiable extension of $\varphi_{2}(t)$ from $\left(t_{k}, t_{k+1}\right)$ to $R$.
Step 4: Let us define yet another function by $\varphi_{\varepsilon}(t)=t_{s}+\frac{\varepsilon}{M+1} \varphi_{2}(t) \forall t \in\left(t_{k}, t_{k+1}\right) \quad$ and $t_{s} \in S$, where $M=\max _{t \in\left[t_{k}, t_{k+1}\right]} \max \left\{\left|\varphi_{2}(t)\right|,\left|\varphi_{2}^{\prime}(t)\right|\right\}$.

Remark 3.4 In addition to Remark 3.3, $\varphi_{\varepsilon}$ fulfils the condition that $\left.\max _{t \in\left[t_{k}, t_{k+1}\right]} \max _{\{}\left|\varphi_{\mathcal{\varepsilon}}(t)\right|,\left|\varphi_{\mathcal{\varepsilon}}^{\prime}(t)\right|\right\}<\mathcal{E}$.

Step 5: Let $0.5>\varepsilon>0$,

$$
h_{\mathcal{E}}(t)=1-\varphi_{\varepsilon}(t)=t-\left(t_{s}+\frac{\varepsilon}{M+1}\left(t-t_{k}\right)^{2}\left(t-t_{k+1}\right)^{2}|t-\rho|^{3} \varphi_{0}(t)\right), \forall t \in\left(t_{k}, t_{k+1}\right)
$$

and $t_{s} \in S$;

then the following can be asserted:

Remark 3.5 Based on Remark 3.4, $h_{\mathcal{\varepsilon}}(t)$ is continuously differentiable in $\left[t_{k}, t_{k+1}\right]$ and its derivative is greater than $1-\varepsilon>0$, hence it is strictly ascending.

Theorem 3.1 The delay $h_{\varepsilon}(t) \leq t$ is a continuous strictly ascending function such that the composite function $f\left(t, x(t), x\left(t-h_{\mathcal{E}}(t)\right)\right)$ has no limit at $\rho$ for $t_{s}<t_{k}<t<t_{k+1}$.

Proof: Note that $t-h_{\varepsilon}(t)=\varphi_{\varepsilon}(t)$ and $\varphi_{\varepsilon}(t)$ passes $t_{s}$ at $t=\rho \pm \frac{1}{k \pi}, 0<k<\infty$. Hence $x\left(t-h_{\mathcal{E}}(t)\right)$ has a jump of the size of $f^{*}\left(t_{s}, x\left(t_{s}\right)\right)$ at each $t=\rho \pm \frac{1}{k \pi}, 0<k<\infty$. Thus $f\left(t, x(t), x\left(t-h_{\mathcal{E}}(t)\right)\right)$ has no limit at $\rho \in\left(t_{k}, t_{k+1}\right)$.

Discussion 3.1 By using continuous delays, the continuity of $f(t, x(t) x(t-h(t)))$ can no longer be relied on as it is currently assumed in the literature since the delay maps the discontinuity of $\mathrm{x}$ at $t_{s}$ into the interval $\left(t_{k}, t_{k+1}\right)$ as an impact of the event at $t_{s}$ on the dynamics of $f(t, x(t) x(t-h(t)))$ at present. In other words, positive increasing continuous functions as delays can be found such that $f(t, x(t) x(t-h(t)))$ is discontinuous/has no limit at some point(s) in $\left(t_{k}, t_{k+1}\right)$.

Furthermore, in this study, the integral equivalence of the formulated system of impulsive delay differential equations is to be obtained for the purpose of analysis of its qualitative properties. In order to achieve this, the following underlying assumptions are employed:

Assumtion 3.1

i) When $t \notin S$, equation (2) reduces to a delay differential equation and solution is obtained from $x^{\prime}(t)=f(t, x(t), \hat{x} \circ(t-\bar{h} \circ \hat{t})), t \in T \mid S ;$

ii) $f$ is continuous in $\Omega$ - an open subset of $T \times R^{(m+1) \times n}$;

iii) For each $t_{k} \in S, x$ is left continuous at $t_{k}$, i.e. $x\left(t_{k}^{-}\right)=x\left(t_{k}\right)$, and there is a jump change at each of these impulse points given by

$$
x\left(t_{k}^{+}\right)=x\left(t_{k}\right)+\Delta x\left(t_{k}\right)=x\left(t_{k}\right)+f^{*}\left(t_{k}, x\left(t_{k}\right)\right) .
$$

iv) After the jump at the moments $t=t_{k}, k=0,1,2, \ldots$, the solution $x(t)$ of equation (2) coincides with the solution $y(t)$ of the initial function problem

$$
\begin{aligned}
& y^{\prime}(t)=f(t, y(t), \hat{y} \circ(t-\bar{h} \circ \hat{t})), t_{k}<t \leq t_{k+1} . \\
& y(t)=x(t), t<t_{k} \text { and } y\left(t_{k}\right)=x\left(t_{k}\right)+f^{*}\left(t_{k}, x\left(t_{k}\right)\right) .
\end{aligned}
$$

v) The function $f(t, x(t) x(t-h(t)))$ is continuous in $x$ for each fixed $t$ in $\left(t_{k}, t_{k+1}\right)$ and measurable in $t$ for each fixed $x$.

To enable us follow the content of this work smoothly, it is necessary to define some basic terms, concepts, notations and lemmas that may be used in the sequel.

Definition 3.1 Let $A \subset B$ be non-empty and $f: B \rightarrow R$, then $\left.f\right|_{A}(x)=f(x), \forall x \in A$ is called the restriction of $f$ to $A$.

Definition 3.2PC[T, $\left.R^{n}\right]=f\left|f: T \rightarrow R^{n}, \quad f\right|_{\left.t_{j}, t_{j+1}\right]} \subset$ $C\left[\left(t_{j}, t_{j+1}\right], R^{n}\right] \Rightarrow \exists f\left(t_{j}+0\right) \in R \forall-\infty<j<\infty$. That is, $f$ restricted to $\left(t_{j}, t_{j+1}\right]$ is continuous.

Definition $3.3 P C\left[[a, b], R^{n}\right]=\left.f\right|_{[a, b]}: f \in P C\left[T, R^{n}\right]$, where $[a, b] \subset T$.

Notation 3.1 Let

i) $[a, b]=\left[a, t_{j_{a}}\right] \cup\left[t_{j_{a}}, t_{j}\right] \cup\left[t_{j}, t_{j+1}\right] \cup\left[t_{j+1}, t_{j+2}\right] \cdots$ $\ldots \cup\left[t_{j_{b}-1}, t_{j_{b}}\right] \cup\left[t_{j_{b}}, b\right]$;

ii) $\quad A_{j_{a}-1}=\left[a, t_{j_{a}}\right], A_{j}=\left[t_{j}, t_{j+1}\right], \forall j_{a} \leq j<j_{b} \quad$ and $A_{j_{b}}=\left[t_{j_{b}}, b\right]$;

iii) $C\left[A_{j}, R^{n}\right]$ denote the set of continuous $R^{n}$-valued functions on the closed and bounded interval $A_{j}$, where $j_{a}-1 \leq j \leq j_{b}$.

Lemma 3.1 If $f \in P C\left[T, R^{n}\right]$ and $[a, b] \subset T$, then 
$\|f\|_{[a, b]}=\sup _{x \in[a, b]}\|f(x)\|$ is a norm and the ordered pair, $\left\{P C\left([a, b], R^{n}\right),\|*\|_{[a, b]}\right\}$ is a Banach space.

Proof:

i) Since $[a, b] \subset T$ is a closed and bounded interval such that $s_{0}<a<b<s_{1}, S_{[a, b]}=[a, b] \cap S$ is a finite set since $\mathrm{S}$ has no condensation point in $[a, b]$. Let $S_{[a, b]}=[a, b] \cap S=\left\{t_{j}\right\}_{j=j_{a-1}}^{j_{b}} \subset[a, b] \subset T \quad$, then $[a, b]=\left[a, t_{j_{a}}\right] \cup\left(\bigcup_{j=j_{a}}^{j_{b}-1}\left(\left[t_{j}, t_{j+1}\right]\right)\right) \cup\left[t_{j_{b}}, b\right]$.

ii) If $a, b \in S$, then $a=t_{j_{a}}$ and $b=t_{j_{b}}$. Hence, $\left[a, t_{j_{a}}\right]=\left\{t_{j_{a}}\right\} \subset\left[t_{j_{a}}, t_{j_{a}+l}\right] \quad$ and $\quad\left[t_{j_{b}}, b\right]=\left\{t_{j_{b}}\right\} \subset$ $\left[t_{j_{b}-1}, t_{j_{b}}\right]$. Otherwise, if $a \notin S$, then $\left[a, t_{j_{a}}\right]$ is a closed bounded interval and $\|f()$.$\| is a continuous function on it.$ If $b \notin S$, then $\left[t_{j_{b}}, b\right]$ is a closed and bounded interval and $\|f()$.$\| has a continuous extension on it by the existing right$ limit $f\left(t_{j_{b}}+0\right)$. Finally, $\|f()$.$\| has a continuous extension$ from $\left(t_{j}, t_{j+1}\right]$ to $\left[t_{j}, t_{j+1}\right]$ by the existence of the right limit $f\left(t_{j}+0\right), \forall j_{a} \leq j<j_{b}$. Thus

$$
\text { iii) }\|f\|_{j_{a}-1}=\sup _{x \in\left[a, t j_{a}\right]}\|f(x)\|
$$

and

$$
\|f\|_{j_{b}}=\sup _{x \in\left[t_{j_{b}}, b\right]}\|f(x)\|
$$

are finite by condition (ii). Again,

$$
\|f\|_{[a, b]}=\sup _{x \in[a, b]}\|f(x)\|=\max _{j_{a}-l<j<j_{b}}\|f\|_{j} .
$$

iv) Since $\quad P C\left[[a, b], R^{n}\right] \subset L_{\infty}\left[[a, b], R^{n}\right] \quad$ and $L_{\infty}\left[[a, b], R^{n}\right]$ is a Banach space with essential sup norm, It follows that $\|\cdot\|_{[a, b]}$ is a norm, where $L_{\infty}\left[[a, b], R^{n}\right]$ is called a Banach space. $f \in L_{\infty}\left[[a, b], R^{n}\right]$ with $\|f\|_{\infty}=0$ $\Rightarrow f=0$ almost everywhere and not everywhere. Hence the real Banach space is the factor space

$$
L_{\infty}\left[[a, b], R^{n}\right] \text { with }\left\{f \mid f \in L_{\infty}\left[[a, b], R^{n}\right],\|f\|_{\infty}=0\right\} .
$$

However,

$$
P C\left[[a, b], R^{n}\right] \cap\left\{f \mid f \in L_{\infty}\left[[a, b], R^{n}\right],\|f\|_{\infty}=0\right\}=\{0\},
$$

hence, the supremum so defined gives a norm on $P C\left[[a, b], R^{n}\right]$ which is a closed linear subspace of $L_{\infty}\left[[a, b], R^{n}\right]$.

Now, let the sequence $\left\{f_{j}\right\}_{j=1}^{\infty} \subset \quad P C\left[[a, b], R^{n}\right] \subset$ $L_{\infty}\left[[a, b], R^{n}\right]$ be convergent to $f \in L_{\infty}\left[[a, b], R^{n}\right]$.

Then from $\|h\|_{[a, b]}=\sup _{x \in[a, b]}\|h(x)\|=$ $\max _{j_{a} \leq j \leq j_{b}}\|h\|_{j} \geq\|h\|_{k}, \forall j_{a}-1 \leq k \leq j_{b} \quad, \quad$ it follows that $f_{j} \rightarrow f \in L_{\infty}\left[[a, b], R^{n}\right]$ for $j \rightarrow \infty$ in $\|\cdot\|_{[a, b]}$ $\Rightarrow f_{j} \rightarrow f \in C_{k}\left[[a, b], R^{n}\right]$ in $\|.\|_{k} \forall j_{a}-1 \leq k \leq j_{b}$

Hence the sequence of functions in $P C\left[[a, b], R^{n}\right]$ is continuous and convergent to $\mathrm{f}$ uniformly in each interval $A_{k}$, and this proves that $\mathrm{f}$ is continuous on these intervals $\forall j_{a}-1 \leq k \leq j_{b}$. Thus $f \in P C\left[[a, b], R^{n}\right]$.

Lemma 3.2 Let $\mathrm{f} \in P C_{1}\left[T, R^{n}\right]$. Then

$$
\left.f\right|_{\left[t_{j}, t_{j+1}\right]} \subset C_{1}\left(\left(t_{j}, t_{j+1}\right], R^{n}\right) \text { and }\left.f\right|_{\left[t_{j}, t_{j+1}\right]}(t)=f\left(t_{j}+0\right)+\int_{t_{j}}^{t} f^{\prime}(s) d s \quad \forall t \in\left(t_{j}, t_{j+1}\right],-\infty<j<\infty .
$$

Proof: This follows from properties of indefinite integrals. Now, to establish the qualitative properties of equations (2) and (4) such as the existence of solution of the initial function problem, the continuous dependence of the solutions on the initial function (4) and stability of the solutions, the integral equivalence of the problem is adopted.

Using Lemma 3.2 and notation (1.1) equation (2) is re-written as:

$$
\bar{x} \circ \hat{t}=x\left(t_{k}\right)+\int_{t_{k}}^{t} f(s, x(s), \hat{x} \circ(\hat{s}-\bar{h} \circ \hat{s})) d s+\sum_{t_{m} \leq t_{k}<t} f^{*}\left(t_{m}, x\left(t_{m}\right)\right)
$$

where $f^{*}\left(t_{m}, x\left(t_{m}\right)\right)=x\left(t_{m}^{+}\right)-x\left(t_{m}\right)$ is the prescribed jump at the impulse point $t_{m} \in S, \forall k=1,2,3, \ldots$ and $t>t_{k}$. With the 
formulation (15) above, some conditions are imposed on $f$ and $x$ to ensure the existence and uniqueness of solutions and the continuous dependence of solutions on the initial function. To obtain the stability of solutions, the problem is re-formulated slightly by separating the linear components of $f$ from the non-linear component as follows:

$$
\left\{\begin{array}{rr}
x^{\prime}(t)=\tilde{f}(x(t), \hat{x} \circ(\hat{t}-\bar{h} \circ \hat{t}))+f_{*}(t, x(t), & \hat{x} \circ(\hat{t}-\bar{h} \circ \hat{t})), \\
\Delta x\left(t_{k}\right)=f^{*}\left(t_{k}, x\left(t_{k}\right)\right), & \forall t \in T \mid S
\end{array}\right.
$$

where $\tilde{f}$ and $f_{*}$ are respectively the linear and non-linear components of $f$. This formulation enables us to develop a scheme for stability of solutions similar to that of Perron. In his work [27], Perron discussed the stability and asymptotic stability of solutions of

$$
x^{\prime}(t)=\tilde{f}(t, x(t))+f_{*}(t, x(t))
$$

with respect to the stability and asymptotic stability of the solution of the homogeneous linear system

$$
y^{\prime}(t)=\tilde{f}(t, y(t))
$$

In line with Perron's formulation, in place of relation (16), the system

$$
\left\{\begin{array}{l}
y^{\prime}(t)=\tilde{f}(t, y(t), \hat{y} \circ(\hat{t}-\bar{h} \circ \hat{t})) \forall t \in T \mid S \\
\Delta y\left(t_{k}\right)=f^{*}\left(t_{k}, y\left(t_{k}\right)\right) \forall t \in S
\end{array}\right.
$$

will be examined subject to the initial condition in equation (4), where $f^{*}\left(t_{k}, y\left(t_{k}\right)\right)$ prescribes the jump at $t_{k}$. Therefore, the integral equivalence of this is:

$$
\bar{y} \circ \hat{t}=y\left(t_{k}\right)+\int_{t_{k}}^{t} \tilde{f}(s, y(s), \hat{y} \circ(\hat{s}-\bar{h} \circ \hat{s})) d s+\sum_{t_{m} \leq t_{k}<t} f^{*}\left(t_{m}, y\left(t_{m}\right)\right) .
$$

\section{Conclusion}

The solutions of impulsive differential equations with the general concept of delays are, however, fundamentally different from those of ordinary differential equations. It is observed that the derivative of the solutions become discontinuous even at non-impulse points and so ceases to be differentiable. Also, the forcing function has no limit even when the delays are strictly ascending and continuously differentiable functions. Here, it has been shown that it is possible to define a continuous ascending delay function whose derivative exists everywhere on an interval $I_{k}$ extendible to $T$, whereas the right-hand side of equation (2) does not have limits at certain other points which are not even impulse points. The integral equivalence of the formulated system of impulsive delay differential equations has also been obtained.

\section{References}

[1] Bereketoglu, H. and Karakoc, F. (2008). Asymptotic, constancy for impulsive delay differential equations. Dynamic Systems and Applications, 17, pp. 71-84.

[2] Dubeau, F. and Karrakchou, J. (2002). State-dependent impulsive delay differential equations, Applied Mathematics Letters, 15, pp. 333-338.

[3] Ballinger, H. G. (1999). Qualitative Theory of impulsive Delay Differential equations, Unpublished $\mathrm{PhD}$ Thesis, University of Waterloo, Canada.

[4] Liu, X. and Ballinger, G. (2002). Existence and continuability of solutions for differential equations with delays and statedependent impulses, Nonlinear Analysis TMA, 51, pp. 633-647.

[5] Oyelami, B., Ale, S. O., Ogidi, J. A., and Onumanyi, P. (2003), Impulsive hiv - 1 model in the presence of antiretroval drugs using $\mathrm{b}$ transform method, Proceedings of African Mathematical Union, 1, pp. 62-76.

[6] Yan, J. (2004), Oscillation properties of a second order impulsive delay differential equation, Comp. and Math. with Applications, 47, pp. 253-258.

[7] Esuabana, I. M. and Abasiekwere, U. A., On stability of first order linear impulsive differential equations, International Journal of Statistics and Applied, Mathematics, Volume 3, Issue 3C, 231-236, 2018.

[8] Abasiekwere, U. A., Esuabana, I. M., Isaac, I. O., Lipcsey, Z, Classification of Non-Oscillatory Solutions of Nonlinear Neutral Delay Impulsive Differential Equations, Global Journal of Science Frontier Research: Mathematics and Decision Sciences (USA), Volume 18, Issue 1, 49-63, 2018, doi:10.17406/GJSFR. 
[9] Abasiekwere, U. A., Esuabana, I. M., Oscillation Theorem for Second Order Neutral Delay Differential Equations with Impulses, International Journal of Mathematics Trends and Technology, Vol. 52 (5), 330-333, 2017, doi:10.14445/22315373/IJMTTV52P548.

[10] Abasiekwere, U. A., Esuabana, I. M., Isaac, I. O., Lipcsey, Z., Existence Theorem For Linear Neutral Impulsive Differential Equations of the Second Order, Communications and Applied Analysis, USA, Vol. 22, No. 2, 135-147, 2018, doi:10.12732/caa.v22i2.1.

[11] Abasiekwere, U. A., Esuabana, I. M., Isaac, I. O., Lipcsey, Z., Oscillations of Second order Impulsive Differential Equations with Advanced Arguments, Global Journal of Science Frontier Research: Mathematics and Decision Sciences (USA), Volume 18, Issue 1, 25-32, 2018, doi:10.17406/GJSFR.

[12] J. A. Ugboh, I. M. Esuabana, Existence and Uniqueness Result for a Class of Impulsive Delay Differential Equations, International Journal of Chemistry, Mathematics and Physics, Vol. 2 (4), 27-32, 2018, doi:10.22161/ijcmp.2.4.1.

[13] Abasiekwere, U. A., Esuabana, I. M., Asymptotic behaviour of nonoscillating solutions of neutral delay differential equations of the second order with impulses, Journal of Mathematical and Computational Science, Vol 8, No 5 (2018), 620-629, doi: $10.28919 / \mathrm{jmcs} / 3765$.

[14] Esuabana, I. M., Abasiekwere, U. A., Ugboh, J. A., Lipcsey, Z. Equivalent Construction of Ordinary Differential Equations from Impulsive System, Academic Journal of Applied Mathematical Sciences, Vol. 4, No. 8, 2018, 77-89.

[15] Bainov, D. D. and Simeonov, P. S. (1995), Impulsive Differential Equations - Asymptotic Properties of the Solutions, World Scientific Pub. Coy. Pte. Ltd, Singapore.

[16] Lakshmikantham, V., Bainov, D. D. and Simeonov, P. S. (1989), Theory of Impulsive Differential Equations, World Scientific Publishing Company Limited, Singapore.

[17] Oyelami, B. O. (1999), On military model for impulsive reinforcement functions using exclusion and marginalization techniques, Nonlinear Analysis, Vol 35, pp. 947-958.
[18] Samaoilenko, A. M. and Perestyuk, J. J. J. (1995), Impulsive Differential Equations, World Scientific, New York.

[19] Bainov, D. D. and Simeonov, P. S. (1989), Systems with Impulse Effects, Stability, Theory and Applications, Ellis Horwood Series in Mathematics and its Applications.

[20] Bainov, D. D. and Simeonov, P. S. (1993), Impulsive Differential Equations - Periodic Solutions and Applications, Longman Scientific and Technical.

[21] Simeonov, P. S. and Bainov, D. D. (1988). Differentiability of solutions of systems with impulse effects with respect to initial data and parameters, Proceedings of Edinburgh Maths Society, 31, pp. 353-368.

[22] Arino, O. and Pituk, M. (2001), More on linear differential systems with small delays, Journal of Differential Equations, Vol 17, pp. 381-407.

[23] Bastinec, J., Diblik, J. and Smarda, Z. (2000), Convergence tests for one scalar differential equation with vanishing delay, Arch. Math. (Brno), 36, pp. 405-414. CDDE Issue.

[24] Oyelami, O. B. and Ale, S. O. (2002). B - transform and its application to fish - hyacinth model, International Journal of Mathematics, Education, Science and Technology, 33 (4), pp. 565-573.

[25] Bainov, D. D. and Stamova, I. M. (1999), Existence, uniqueness and continuability of solutions of differentialdifference equations, Journal of Applied Mathematics and Stochastic Analysis, 12: pp. 293-300.

[26] Gopalsamy, K. (1992), Stability and Oscillation in Delay Differential Equations of Population Dynamics, Kluwer Academics, Dordrecht.

[27] Coddington, A. E. and Levinson, N. (1955), Theory of Ordinary Differential Equations, McGraw-Hill Book Company, New York. 